\title{
Prevalence of Superior Semicircular Canal Dehiscence on High- Resolution CT Imaging in Patients without Vestibular or Auditory Abnormalities
}

\author{
(D) A.W. Berning, ${ }^{\text {K. }}$. Arani, and (D) B.F. Branstetter, IV
}

\begin{abstract}
BACKGROUND AND PURPOSE: Prior studies of radiologic superior semicircular canal dehiscence have suggested that CT may overcall dehiscence. However, many of those studies were performed before the advent of multichannel helical CT. Furthermore, there are limited data investigating the prevalence of radiologic superior semicircular canal dehiscence in asymptomatic individuals. The purpose of this study was to determine the rate of radiologic superior semicircular canal dehiscence in an asymptomatic population using 64-channel helical CT.
\end{abstract}

MATERIALS AND METHODS: We retrospectively enrolled 500 consecutive adult patients without symptoms of superior semicircular canal dehiscence who had undergone CT of the temporal bones in the emergency department of a tertiary academic center between February 2012 and June 2017. The superior semicircular canals were evaluated bilaterally by 2 dedicated head and neck radiologists and subjectively classified as either dehiscent or nondehiscent. A secondary group of 110 scans of patients with symptoms consistent with superior semicircular canal dehiscence was similarly interpreted, and the rate of radiologic superior semicircular canal dehiscence was calculated for each group.

RESULTS: Ten of the 500 asymptomatic patients (2.0\%; $95 \% \mathrm{Cl}, 1.1 \%-3.6 \%)$ had CT evidence of superior semicircular canal dehiscence, compared with 15 of 110 symptomatic patients (13.6\%; $95 \% \mathrm{Cl}, 7.8 \%-21.5 \%)$. There was excellent interobserver agreement $(\kappa=0.91)$.

CONCLUSIONS: Only $2 \%$ of asymptomatic patients had radiologic evidence of superior semicircular canal dehiscence on 64 -detector row helical CT. This is markedly lower than previous reports and approaches the postmortem rate of asymptomatic superior semicircular canal dehiscence. We therefore recommend that asymptomatic patients with CT evidence of superior semicircular canal dehiscence undergo audiologic evaluation because the rate of false-positive scans is extremely low.

ABBREVIATION: SSCD = superior semicircular canal dehiscence

$F^{\prime}$ ollowing its initial description in 1998, superior semicircular canal dehiscence (SSCD) syndrome has become recognized as a frequent cause of auditory and vestibular abnormalities. ${ }^{1}$ The syndrome is associated with variable patient presentations including vertigo, conductive hearing loss, autophony, tinnitus, sound-induced vertigo (Tullio phenomenon), and other related symptoms. ${ }^{2}$ In SSCD, these symptoms appear in the setting of dehiscence of the bony roof of the superior semicircular canal, which is thought to cause symptoms by creating an abnormal pathway (a so-called "third window") for sound and pressure conduction. ${ }^{3}$ The suspected diag-

Received September 15, 2018; accepted after revision January 28, 2019.

From the University of Pittsburgh School of Medicine (A.W.B.) and Departments of Radiology (K.A., B.F.B.) and Otolaryngology (B.F.B.), University of Pittsburgh Medical Center, Pittsburgh, Pennsylvania.

Please address correspondence to Aric W. Berning, MD, S530 Scaife Hall, 3550 Terrace St, Pittsburgh, PA 15213; e-mail: aric.berning@pitt.edu

http://dx.doi.org/10.3174/ajnr.A5999 nosis of SSCD is aided by audiometry and vestibular testing and confirmed by high-resolution CT demonstrating dehiscence on coronally reformatted images. ${ }^{4}$ Symptoms may be treated with vestibular sedation or surgical repair. ${ }^{5}$

Cadaveric studies have demonstrated dehiscence of the superior semicircular canal in $0.5 \%$ of specimens, and near-dehiscence (defined as a bone thickness of $\leq 0.1 \mathrm{~mm}$ ) in an additional 1.4\%. ${ }^{6}$ In contrast, imaging studies of temporal bone CT have reported rates of radiologic dehiscence between $3.0 \%$ and $9.0 \%$, suggesting that CT may overcall dehiscence. ${ }^{7-11}$ However, many of these studies are limited by small sample sizes, and many were conducted before the advent of multislice helical CT. Furthermore, there are limited data directly describing the prevalence of dehiscence in individuals without symptoms of SSCD (so-called "incidental radiologic dehiscence"). These data would be useful to guide management of patients in whom SSCD is incidentally detected on CT.

AJNR Am J Neuroradiol 40:709-12 Apr 2019 www.ajnr.org 
The present study, therefore, attempts to address this gap in the literature by assessing the prevalence of radiologic SSCD in patients without auditory or vestibular symptoms, using current high-resolution CT protocols. The primary aim of this study was to report the prevalence of SSCD in this asymptomatic population to guide clinical management of these patients. A secondary analysis was to compare the rate of incidental radiologic SSCD with the rate of radiologic SSCD in patients presenting with symptoms associated with the diagnosis.

\section{MATERIALS AND METHODS}

This study received ethics approval from our institutional review board.

\section{Subjects}

We retrospectively reviewed the records of patients presenting to the emergency department of a large, tertiary care, university hospital between February 2012 and August 2017. Patients were included if they were 18 years of age or older and had undergone CT of the temporal bone. Patients were excluded if the CT was not conducted using the appropriate high-resolution temporal bone protocol or if the semicircular canal could not be visualized due to pathology or artifacts. To prevent interpretation bias, we initially included CT scans regardless of patient clinical history. After interpretation of the scans, subjects were sorted into 2 groups based on provided indications for imaging: those without symptoms consistent with SSCD (asymptomatic patients) and those with symptoms consistent with SSCD (symptomatic patients). Vertigo, hearing loss, dizziness, and tinnitus were considered symptoms consistent with SSCD in this study. On the basis of an a priori power analysis assuming a prevalence of $3.0 \%$ and confidence interval half-widths of $1.5 \%$, we consecutively enrolled patients until we reached 500 asymptomatic patients.

\section{Imaging Protocol and Techniques}

CT was acquired using LightSpeed 64-channel CT scanners (GE Healthcare, Milwaukee, Wisconsin) with a slice thickness of 0.63 $\mathrm{mm}$, spacing of $0.375 \mathrm{~mm}, 120 \mathrm{kV}$ (peak), $195 \mathrm{~mA}$, pitch of 0.53 , bone kernel, and matrix of $512 \times 512$. Images were obtained in the axial plane with coronal reformats (1-mm-thick, 1-mm spacing) because prior studies have demonstrated that these views are sufficient to accurately identify dehiscence compared with reformats in the planes of Stenver and Poschl. ${ }^{4}$

\section{Data Collection}

Patient age, sex, and indication for imaging were recorded. Patients' CT scans were independently reviewed by 2 fellowshiptrained, dedicated head and neck radiologists who were blinded to the clinical history of the patient. For each scan, the superior semicircular canals were evaluated bilaterally, and the bone overlying the canal was subjectively classified as either intact (Figs 1 and 2) or dehiscent (Fig 3). Very thin bone overlying the superior semicircular canal was not considered dehiscent (Fig 4). After we assessed interobserver reliability, discrepancies between observers were resolved by consensus to establish descriptive statistics.

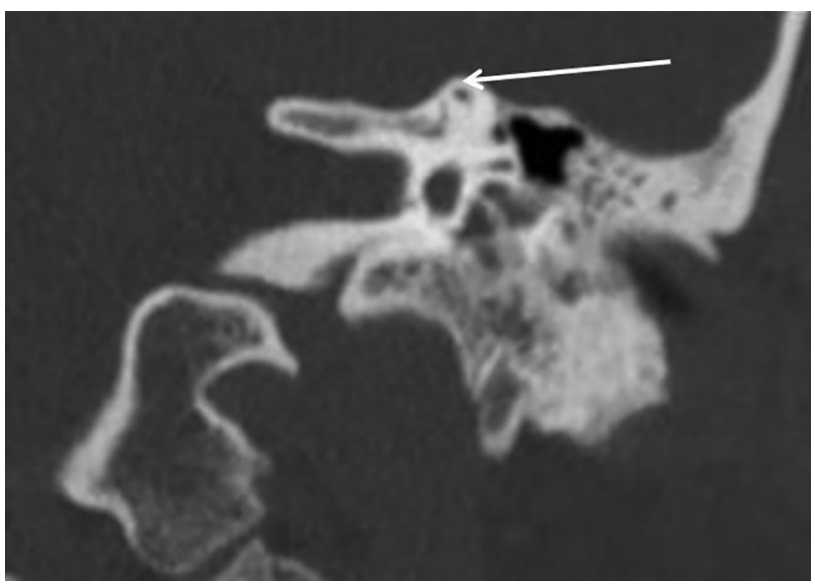

FIG 1. Intact superior semicircular canal. Coronally reformatted CT of the temporal bone demonstrates an intact roof of the superior semicircular canal (arrow).

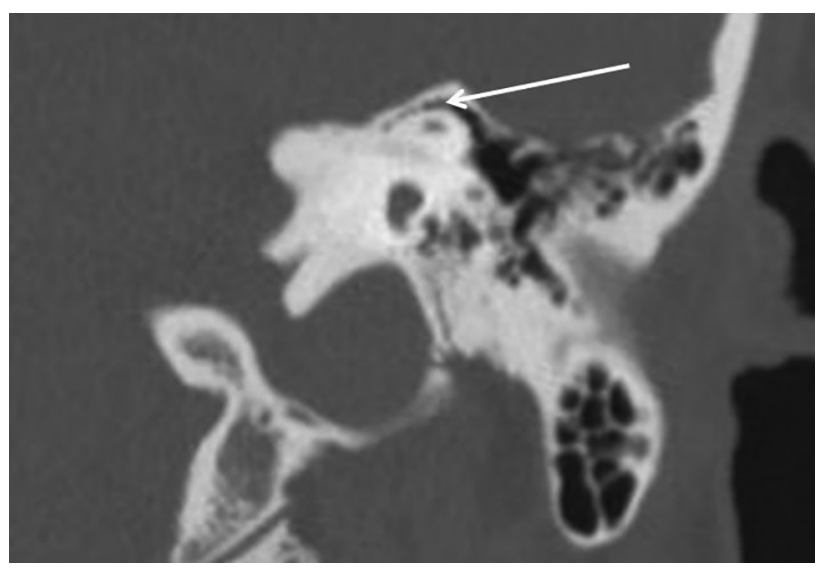

FIG 2. Air cells overlying the superior semicircular canal. Coronally reformatted CT of the temporal bone demonstrates an intact superior semicircular canal (arrow) with overlying air cells. When air cells are present, the canal should be classified as nondehiscent, regardless of the thickness of bone covering the canal or underlying the dura.

\section{Statistical Analysis}

The prevalence of radiologic SSCD in both asymptomatic and symptomatic patient groups was calculated, along with $95 \%$ confidence intervals using the Newcombe method for extreme percentages. ${ }^{12}$ Comparisons between patient groups were conducted using the Fisher exact test for categoric variables and the Student $t$ test for continuous variables. Interrater reliability was calculated using the Cohen $\kappa$ statistic. A $P$ value of .05 was the threshold for all tests of significance. Statistical calculations were performed using the SPSS software package, Version 23 (IBM, Armonk, New York).

\section{RESULTS}

A total of 681 patient records were evaluated for possible inclusion. Of these, 24 were excluded due to incomplete data, and 33 were excluded for protocol errors. An additional 14 were excluded for inability to evaluate the semicircular canals due to motion artifacts or overlying pathology. One hundred ten patients were excluded from the primary analysis due to symptoms consistent with SSCD, leaving 500 asymptomatic patients for prevalence cal- 


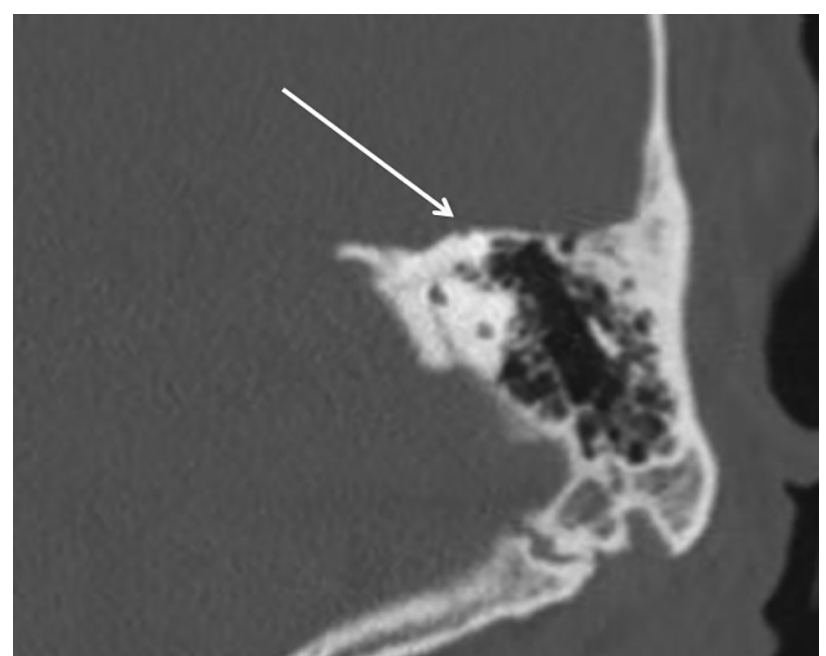

FIG 3. A thin-but-intact superior semicircular canal. Coronally reformatted CT of the temporal bone demonstrates very thin bone overlying the superior semicircular canal (arrow). This quantity of bone should be classified as intact in patients with and without suggestive symptoms.

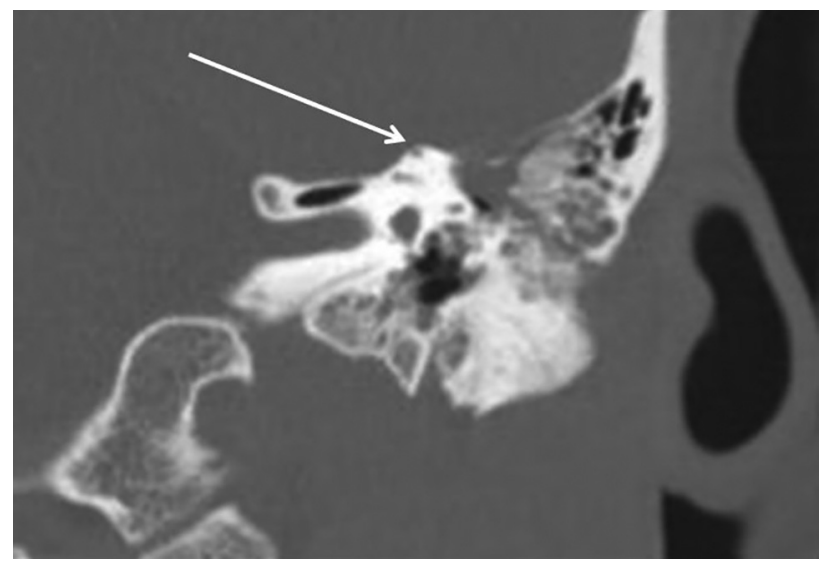

FIG 4. Dehiscent superior semicircular canal. Coronally reformatted $\mathrm{CT}$ of the temporal bone demonstrates dehiscence of the roof of the superior semicircular canal (arrow).

Table 1: Asymptomatic $(n=500)$ patient characteristics and indications for CT

\begin{tabular}{lc}
\hline Characteristics/Indications & \\
\hline Mean age (range) (yr) & $50.7 \pm 15.8(19-87)$ \\
No. male (\%) & $336(67.2 \%)$ \\
Imaging indication (No.) (\%) & \\
Trauma & $368(77.6 \%)$ \\
Mass & $45(9.0 \%)$ \\
Infection & $33(6.6 \%)$ \\
Pain & $26(5.2 \%)$ \\
CSF leak/cephalocele & $14(2.8 \%)$ \\
Cranial nerve palsy & $5(1.0 \%)$ \\
Otosclerosis & $4(0.8 \%)$ \\
Surgical planning & $3(0.6 \%)$ \\
Radionecrosis & $2(0.4 \%)$ \\
\hline
\end{tabular}

culation. Patient demographics and indications for imaging are summarized in Tables 1 and 2.

Ten of the 500 asymptomatic patients had CT evidence of $\operatorname{SSCD}(2.0 \%$; 95\% CI, 1.0\%-3.8\%). Of these 10 patients, 7 were imaged for trauma; 2 , for evaluation of a mass; and 1, for evalua-
Table 2: Symptomatic $(n=110)$ patient characteristics and indications for $\mathrm{CT}$

\begin{tabular}{lc}
\hline Characteristics/Indications & \\
\hline Mean age (range) (yr) & $47.6 \pm 18.9(18-100)$ \\
No. male (\%) & $41(37.3 \%)$ \\
Imaging indication (No.) (\%) & \\
$\quad$ Dizziness & $62(56.4 \%)$ \\
Suspected SSCD & $31(28.1 \%)$ \\
Hearing loss & $23(20.1 \%)$ \\
Vertigo & $6(5.5 \%)$ \\
Tinnitus & $6(5.5 \%)$ \\
\hline
\end{tabular}

tion of infection. Three patients, all imaged for trauma, demonstrated bilateral SSCD. The most common indication for imaging in the asymptomatic group was trauma; 7 of the 368 patients with this indication were found to have radiologic dehiscence $(1.9 \%$; 95\% CI, 0.8\%-4.0\%).

In contrast, radiologic SSCD was detected in 15 of the 110 symptomatic patients $(13.6 \%$; $95 \% \mathrm{CI}, 8.1 \%-21.8 \%)$. Two of the symptomatic patients demonstrated bilateral dehiscence. The rate of bilateral dehiscence did not differ significantly between groups $(P=.22)$. The difference in prevalence of radiologic SSCD between the 2 groups was statistically significant $(P<.001)$.

For 31 of the 110 symptomatic patients, the provided indication for CT was evaluation of suspected SSCD. Ten patients in this subgroup demonstrated radiologic SSCD (32.3\%; 95\% CI, $17.3 \%-51.5 \%)$. Five of the remaining 79 symptomatic patients demonstrated radiologic SSCD (6.3\%; 95\% CI, 2.4\%-14.8\%). The difference in the rate of radiologic SSCD was statistically significant both among these subgroups of symptomatic patients $(P<.001)$ and between each subgroup and the group of asymptomatic patients $(P<.05)$.

There was excellent interrater agreement between the $2 \mathrm{ob}-$ servers $(\kappa=0.91)$, with disagreement on only 5 of the 1220 temporal bones that were evaluated $(0.4 \%)$.

\section{DISCUSSION}

In a retrospective study of 500 patients without symptoms of SSCD, radiologic evidence of dehiscence was detected in $2.0 \%$ of cases, with excellent agreement between expert observers. This was significantly lower than the $13.6 \%$ prevalence of dehiscence in a similar group of 110 patients with symptoms that might be seen in SSCD.

This prevalence of $2 \%$ is notably lower than the prevalence of $9.0 \%$ reported by Williamson et $\mathrm{al}^{11}{ }^{11}$ which has been cited in 2 recent comprehensive review articles on SSCD. ${ }^{2,13}$ Most important, this previous work was conducted before the advent of 64detector row helical CT, which may explain the higher prevalence reported in earlier studies. More recent studies with contemporary CT protocols have reported a lower prevalence of SSCD; however, these studies are limited by relatively small sample sizes or include patients with symptoms consistent with SSCD and thus do not provide reliable estimates of the prevalence of SSCD in asymptomatic individuals. For example, Nadgir et $\mathrm{al}^{9}$ reported dehiscence of $7.8 \%$ in a study of 306 CT scans, but the focus of the study was to categorize the prevalence of SSCD by age and included patients with a variety of vestibular and auditory symptoms. Similarly, Crovetto et $\mathrm{al}^{14}$ reported radiologic SSCD in $3.6 \%$ of studied ears but did not exclude symptomatic patients. 
Our results are most consistent with a recent study by KloppDutote et al, ${ }^{15}$ in which radiologic SSCD was detected in $1.7 \%$ of a population of 180 patients, but it is unclear whether this population included patients with otologic symptoms and may be limited by a small sample size.

In an anatomic study of 1000 temporal bones from 596 individuals, Carey et $\mathrm{al}^{6}$ identified complete dehiscence in $0.5 \%$ of specimens. An additional $1.4 \%$ of specimens was noted to have markedly thin $(\leq 0.1 \mathrm{~mm})$ bone overlying the superior semicircular canal. Individuals with such thin coverings may appear dehiscent even on high-resolution CT scans; thus, our results correlate well with the reported postmortem rate of dehiscence.

The results of our study suggest that incidental radiologic SSCD is rare in individuals without clinical symptoms of auditory or vestibular dysfunction. Given that the rate of false-positive scans is low, we recommend that patients in whom radiologic dehiscence is discovered incidentally on CT undergo audiologic evaluation to screen for SSCD.

The present study is limited by its retrospective design and incomplete access to patient data. The clinical syndrome of SSCD is highly variable, and it is possible that patients categorized as asymptomatic in our study may have otologic symptoms that were not reported at their emergency department presentation and thus have been erroneously classified as asymptomatic. Also due to the retrospective study design, we are unable to determine whether patients subsequently developed otologic symptoms following their initial emergency department presentation. Furthermore, a multitude of entities besides SSCD can present with symptoms of hearing loss, vertigo, tinnitus, and dizziness.

Our population of asymptomatic patients had a male predominance, likely due to the high number of trauma cases in our emergency department population. This male predominance was not mirrored in our concurrent sample of symptomatic patients. However, SSCD is not known to have a sex predilection. Our results may not be generalizable to examinations performed on scanners with fewer than 64 channels.

Furthermore, our inclusion of patients evaluated for suspected SSCD biases the reported prevalence of SSCD in the larger group of symptomatic patients. Of the 15 cases of radiologic SSCD in our symptomatic population, 10 belonged to this subgroup, which demonstrated a prevalence of radiologic SSCD of 32.3\%. Excluding patients who underwent CT for evaluation of suspected SSCD lowers the prevalence of radiologic SSCD in the symptomatic group to $6.3 \%$, which remains significantly higher than the rate of $2 \%$ detected in the asymptomatic group. Our reported prevalence of SSCD in symptomatic patients should be cautiously interpreted due to its small sample size; however, our reported prevalence of SSCD in asymptomatic patients does not have this limitation.

\section{CONCLUSIONS}

There is radiologic evidence of SSCD in $2 \%$ of patients without clinical symptoms of auditory or vestibular dysfunction. Given this low rate of false-positives, we recommend audiologic evaluation for patients in whom SSCD is incidentally discovered on CT imaging.

\section{REFERENCES}

1. Minor LB, Solomon D, Zinreich JS, et al. Sound- and/or pressureinduced vertigo due to bone dehiscence of the superior semicircular canal. Arch Otolaryngol Head Neck Surg 1998;124:249-58 CrossRef Medline

2. Mau C, Kamal N, Badeti S, et al. Superior semicircular canal dehiscence: diagnosis and management. J Clin Neurosci 2018;48: 58-65 CrossRef Medline

3. Rosowski JJ, Songer JE, Nakajima HH, et al. Clinical, experimental, and theoretical investigations of the effect of superior semicircular canal dehiscence on hearing mechanisms. Otol Neurotol 2004;25: 323-32 CrossRef Medline

4. Branstetter BF 4th, Harrigal C, Escott EJ, et al. Superior semicircular canal dehiscence: oblique reformatted CT images for diagnosis. $R a$ diology 2006;238:938-42 CrossRef Medline

5. Minor LB. Clinical manifestations of superior semicircular canal dehiscence. Laryngoscope 2005;115:1717-27 CrossRef Medline

6. Carey JP, Minor LB, Nager GT. Dehiscence or thinning of bone overlying the superior semicircular canal in a temporal bone survey. Arch Otolaryngol Head Neck Surg 2000;126:137-47 CrossRef Medline

7. Gartrell BC, Gentry LR, Kennedy TA, et al. Radiographic features of superior semicircular canal dehiscence in the setting of chronic ear disease. Otol Neurotol 2014;35:91-96 CrossRef Medline

8. Masaki $Y$. The prevalence of superior canal dehiscence syndrome as assessed by temporal bone computed tomography imaging. Acta Otolaryngol 2011;131:258-62 CrossRef Medline

9. Nadgir RN, Ozonoff A, Devaiah AK, et al. Superior semicircular canal dehiscence: congenital or acquired condition? AJNR Am J Neuroradiol 2011;32:947-49 CrossRef Medline

10. Stimmer H, Hamann KF, Zeiter S, et al. Semicircular canal dehiscence in HR multislice computed tomography: distribution, frequency, and clinical relevance. Eur Arch Otorhinolaryngol 2012;269: 475-80 CrossRef Medline

11. Williamson RA, Vrabec JT, Coker NJ, et al. Coronal computed tomography prevalence of superior semicircular canal dehiscence. Otolaryngol Head Neck Surg 2003;129:481-89 CrossRef Medline

12. Newcombe RG. Statistical applications in orthodontics, Part II: confidence intervals for proportions and their differences. J Orthod 2000;27:339-40 CrossRef Medline

13. Ward BK, Carey JP, Minor LB. Superior canal dehiscence syndrome: lessons from the first 20 years. Front Neurol 2017;8:177 CrossRef Medline

14. Crovetto M, Whyte J, Rodriguez OM, et al. Anatomo-radiological study of the superior semicircular canal dehiscence: radiological considerations of superior and posterior semicircular canals. Eur J Radiol 2010;76:167-72 CrossRef Medline

15. Klopp-Dutote N, Kolski C, Biet A, et al. A radiologic and anatomic study of the superior semicircular canal. Eur Ann Otorhinolaryngol Head Neck Dis 2016;133:91-94 CrossRef Medline 
n the article "Prevalence of Superior Semicircular Canal Dehiscence on High-Resolution CT Imaging in Patients without Vestibular or Auditory Abnormalities” by A.W. Berning, K. Arani, and B.F. Branstetter, IV (AJNR Am J Neuroradiol 2019;40:709-12), the images and legends for Figs 3 and 4 were incorrectly matched. They should appear as shown below. The authors regret the error.

http://dx.doi.org/10.3174/ajnr.A6082

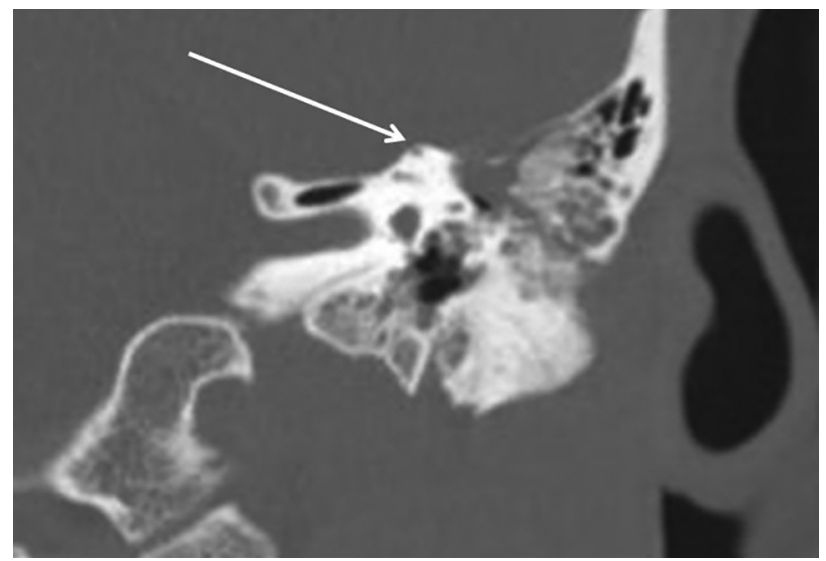

FIG 3. A thin-but-intact superior semicircular canal. Coronally reformatted CT of the temporal bone demonstrates very thin bone overlying the superior semicircular canal (arrow). This quantity of bone should be classified as intact in patients with and without suggestive symptoms.

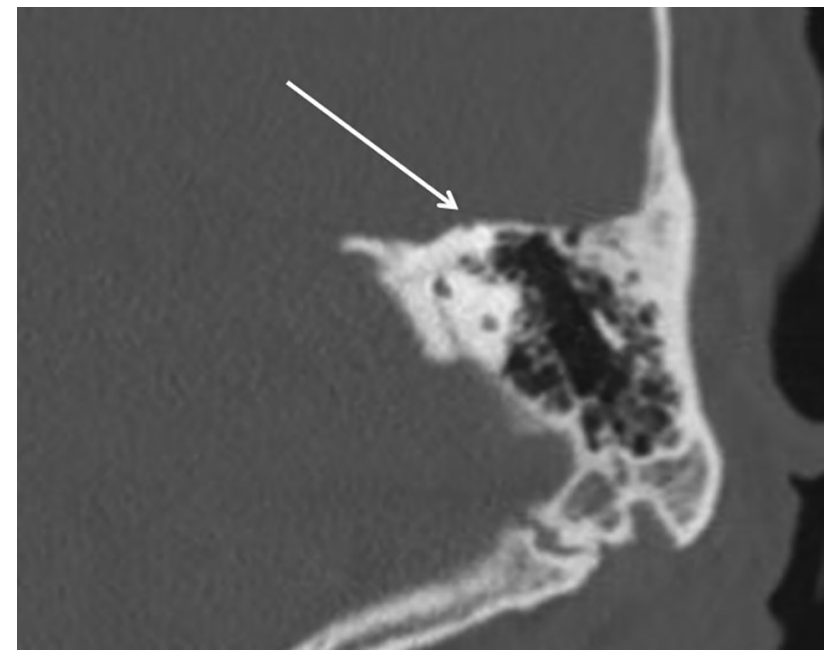

FIG 4. Dehiscent superior semicircular canal. Coronally reformatted $\mathrm{CT}$ of the temporal bone demonstrates dehiscence of the roof of the superior semicircular canal (arrow). 